\title{
Patients with suspected acute coronary syndrome in a university hospital emergency department: an observational study Ulf Ekelund*1,2, Hans-Jörgen Nilsson ${ }^{1}$, Attila Frigyesi ${ }^{1}$ and Ole Torffvit ${ }^{1}$
}

Address: ${ }^{1}$ Department of Medicine, Lund University, Lund, Sweden and 2Department of Physiological Sciences, Lund University, Lund, Sweden

E-mail: Ulf Ekelund* - ulf.ekelund@mphy.lu.se; Hans-Jörgen Nilsson - hagnar33@hotmail.com; Attila Frigyesi - attila@maths.lth.se; Ole Torffvit - ole.torffvit@med.lu.se

${ }^{*}$ Corresponding author

Published: 3 October 2002

BMC Emergency Medicine 2002, 2:I
Received: 28 June 2002

Accepted: 3 October 2002

This article is available from: http://www.biomedcentral.com/I47I-227X/2/I

(c) 2002 Ekelund et al; licensee BioMed Central Ltd. This article is published in Open Access: verbatim copying and redistribution of this article are permitted in all media for any purpose, provided this notice is preserved along with the article's original URL.

Keywords: Acute coronary syndrome, chest pain, emergency department, outcome

\begin{abstract}
Background: It is widely considered that improved diagnostics in suspected acute coronary syndrome (ACS) are needed. To help clarify the current situation and the improvement potential, we analyzed characteristics, disposition and outcome among patients with suspected ACS at a university hospital emergency department (ED).
\end{abstract}

Methods: 157 consecutive patients with symptoms of ACS were included at the ED during 10 days. Risk of ACS was estimated in the ED for each patient based on history, physical examination and ECG by assigning them to one of four risk categories; I (obvious myocardial infarction, MI), II (strong suspicion of ACS), III (vague suspicion of ACS), and IV (no suspicion of ACS).

Results: 4, 17, 29 and $50 \%$ of the patients were allocated to risk categories I-IV respectively. 74 patients $(47 \%)$ were hospitalized but only $19(26 \%)$ had ACS as the discharge diagnose. In risk categories I-IV, ACS rates were 100,37, 12 and $0 \%$, respectively. Of those admitted without ACS, at least $37 \%$ could probably, given perfect ED diagnostics, have been immediately discharged. 83 patients were discharged from the ED, and among them there were no hospitalizations for ACS or cardiac mortality at 6 months. Only about three patients per $24 \mathrm{~h}$ were considered eligible for a potential ED chest pain unit.

Conclusions: Almost $75 \%$ of the patients hospitalized with suspected ACS did not have it, and some $40 \%$ of these patients could probably, given perfect immediate diagnostics, have been managed as outpatients. The potential for diagnostic improvement in the ED seems large.

\section{Background}

Patients with symptoms of acute myocardial infarction (AMI) or unstable angina pectoris (UA), i.e. an acute coronary syndrome (ACS), are very common in emergency departments (EDs). Because of diagnostic difficulties, a certain "overadmission" to in-hospital care for suspected
ACS is usually accepted. The size of this overadmission is generally unknown, despite its negative influence on hospital efficiency and resource utilization. In order to reduce unnecessary admission, to optimize disposition of admitted patients and to decrease cost, chest pain units (CPUs) with risk-based diagnostic protocols have been estab- 
lished in many hospitals, primarily in the United States $[1,2]$. These CPUs have been shown to increase the efficiency of patient evaluation with a quality similar to that of traditional EDs $[3,4]$.

For hospitals with traditional EDs, the benefits of establishing a CPU depend on the quality and outcome of current management, and on patient volume and risk spectrum. Such data are relatively rare, but have been reported from the 1980's from Gothenburg, Sweden [5,6], and from the mid-1990's from hospitals in the United States $[7,8]$. The quality of traditional patient management is constantly improving however, with therapeutic and diagnostic advances such as new antithrombotic drugs [9], ECG decision support software, remote ECG analysis [10], and the use of new combinations of blood tests for myocardial injury $[11,12]$. With generally improved cardiac care, the patient and risk spectrum in the ED are likely also continuously changing. Therefore, in order to plan and implement improved diagnostic strategies in suspected ACS, new and current data are clearly needed.

This study aimed to analyze current characteristics, disposition and outcome of patients with suspected ACS in a traditional ED, and to explore the basis for establishing a CPU. We will report that almost three out of every four patients hospitalized with suspected ACS did not have it, and that some four out of every ten admitted patients could, given perfect immediate diagnostics, have been treated as outpatients.

\section{Methods \\ Study design}

Consecutive patients presenting with symptoms compatible with an ACS were prospectively included during 10 days from September 26 to October 6, 2000. To reflect the true clinical spectrum of patients, a slightly modified version of the IMIR study criteria [13] was used for inclusion. Thus, all patients presenting with chest pain or discomfort, dyspnea without obvious pulmonary disease, acute heart failure, arrhythmia of possible ischemic origin and suspected cardiac syncope were included. Informed consent was obtained from all study patients.

\section{Study setting and population}

Lund University Hospital is a 1200 bed institution with fully public financing that serves a population of some 250,000, has a cardiac intensive care unit (CICU) with 19 beds and an intermediate care ward (ICW) with ECG monitoring at 16 beds. Problems with high bed occupancy have been common in recent years. Both balloon angioplasty (PTCA) and coronary bypass surgery (CABG) are offered 24 hours/day, and ECGs are regularly transmitted from ambulances to the CICU to start thrombolysis or to prepare for direct PTCA on arrival. A traditional ED is in operation with approximately 50 patients per day with problems related to internal medicine. There is no chest pain unit and no systematic diagnostic protocol for patients with suspected ACS. The Siemens Megacart ECG recorders (Siemens-Elema, Solna, Sweden) have the latest Siemens GRI decision support software installed. Serum CKMB mass and Troponin T tests are used for detection of myocardial injury and are available 24 hours.

\section{Study protocol}

Inclusion was performed by the primary assessing nurse and retrospectively reevaluated by one of the investigators (UE). Risk factors and previous cardiovascular disease were noted on a standard form. Data missing on the forms were collected via patient records or, if ambiguous, via telephone interviews with the patients. To be able to compare initial assessment and outcome, physicians on duty were asked to assign each patient to one of four risk categories based on the suspicion of ACS (modified from [5]) after history and physical examination, but before blood tests:

I. Obvious AMI. Typical symptoms and ST-elevation with or without Q-waves on the ECG, or LBBB not known to be old.

II. Strong suspicion of ACS. a. Typical symptoms without ST-elevation or Q-wave. $b$. Atypical symptoms with ST-T changes or LBBB not previously observed. $c$. History of unstable angina regardless of ECG. $d$. Acute heart failure or hypotension regardless of ECG. $e$. VT/VF or AV-block III.

III. Vague suspicion of ACS. Unclear symptoms and history, normal or non-ischemic ECG.

IV. No suspicion of ACS. a. No suspicion of ischemic heart disease. $b$. Stable angina pectoris.

Eligibility for a potential CPU was estimated among low to moderate risk patients (see Results) with the following commonly used [3] exclusion criteria: tachy- (>110 bpm) or brady-arrhythmia $(<40 \mathrm{bpm})$ of any sort, bilateral rales above lung bases (as a sign of heart failure), and inability to perform our standard bicycle ergometry stress test on the basis of muscle, joint or motor nerve problems.

\section{Measurements}

Patient characteristics in the forms of presenting symptoms, risk factors and cardiovascular history were recorded, as well as the physician's immediate risk estimate, the patient's primary in-hospital bed assignment and the discharge diagnosis. In the immediately discharged, hospitalizations for ACS within 6 months, cardiac ischemic events (AMI and revascularisation) and mortality were monitored by reviewing medical records and by tele- 
Table I: Characteristics of I57 consecutive patients with suspected ACS in the ED of Lund University Hospital and comparison with previous surveys in Gothenburg [5] and the USA [7].

\begin{tabular}{|c|c|c|c|c|}
\hline & & $\%$ & $\begin{array}{c}\text { Karlson et al. I99I, } \\
n=7157\end{array}$ & $\begin{array}{c}\text { Pope et al. 1998, } \\
n=10700\end{array}$ \\
\hline \multirow[t]{5}{*}{ Pain/discomfort, localisation } & Chest pain/discomfort & 80 & $93 *$ & 76 \\
\hline & Left arm & 34 & & \\
\hline & Pain/discomfort elsewhere & 30 & & \\
\hline & Pain/discomfort in ED & 61 & & \\
\hline & Other reason for inclusion & 20 & & \\
\hline \multirow[t]{6}{*}{ Risk factors } & Family history of IHD & 46 & & \\
\hline & Diabetes & 12 & 8 & $21^{*}$ \\
\hline & Smoking & 17 & $33 *$ & \\
\hline & Treated hypercholesterolemia & 28 & & \\
\hline & Overweight (BMI>25) & 34 & & \\
\hline & Hypertension & 32 & $24 *$ & \\
\hline \multirow[t]{6}{*}{ Cardiovascular history } & Angina Pectoris & 42 & 39 & 37 \\
\hline & Myocardial infarction & 32 & $24 *$ & 26 \\
\hline & CABG/PTCA & 25 & & \\
\hline & Intermittent claudication & 5 & & \\
\hline & Stroke & 8 & & \\
\hline & Heart failure & 12 & $18 *$ & \\
\hline
\end{tabular}

$* \mathrm{P}<0.05$ compared with the present data (z-test).

phone calls to each patient. A diagnosis of AMI was made at a CKMB serum level $>10 \mu \mathrm{g} / \mathrm{l}$ or a Troponin $\mathrm{T}$ serum level $>0.06 \mu \mathrm{g} / \mathrm{l}$ in the absence of renal failure, and a typical rise and fall pattern on serial blood testing. Both analyses were performed with sandwich immunoassays using double monoclonal antibodies. For the results to reflect the quality of everyday medical service, there was no retrospective review of discharge diagnoses or risk classification, unless obviously wrong.

\section{Data analysis and statistics}

In the final analysis, less than $5 \%$ of all data were missing. Data are presented as means \pm SEM. For statistical comparison, Student's t-test was used except when stated otherwise. Differences were considered significant at $\mathrm{P}<0.05$.

\section{Results}

One hundred and fifty-seven patients with chest pain or other symptoms suggestive of ACS were included during the study period, making up 33\% of all those presenting at the ED with internal medicine problems. The included women $(\mathrm{n}=90)$ were on average slightly older than the men $(n=67), 64.4 \pm 2.1$ vs. $58.1 \pm 2.1$ years $(p=0.04)$. Symptoms, risk factors and cardiovascular history are given in table 1 . Eighty percent had chest pain or discomfort, $61 \%$ had ongoing symptoms in the ED, $42 \%$ and $32 \%$ had previously established ischemic heart disease in the forms of angina pectoris and AMI respectively, and one out of four had a previous PTCA or CABG. Overweight (body mass index >25) was equally common in men and women (34\%).

Physicians' risk estimates after history, physical examination and ECG, and admittance rates are shown in table 2. At that point in time, a suspicion of ACS remained in $50 \%$ of the patients. The higher the judged risk of ACS, the older the average patient.

Forty-seven percent of all included patients were admitted, $43 \%$ of the men and $53 \%$ of the women (n.s.). Primary in-hospital destinations, PTCA rates within 30 days and diagnoses at discharge in the different risk categories are shown in table 3 . In those admitted, there were no significant differences in the discharge diagnoses between men and women. Average time to discharge was $3.5 \pm 0.4$ days (median 2.0 days), and in categories I-IV hospital stays were $7.0 \pm 1.4,4.2 \pm 0.8,2.4 \pm 0.4$ and $3.0 \pm 0.9$ days, respectively.

\section{Patients with ACS as the discharge diagnose}

Of the 74 patients admitted, only 19 (26\%) were diagnosed with an AMI or UA, i.e. an ACS (table 3). Of these, 4 had Q-wave AMI, 7 non-Q-wave AMI (NQMI) and 8 UA. In risk categories I-III, ACS rates among the admitted patients were $100 \%, 37 \%$ and $12 \%$ respectively (table 3 ). Of those admitted to the CICU $(n=18)$, the ICW $(n=51)$ and a regular ward $(n=5), 79 \%, 8 \%$ and $20 \%$ had ACS, respectively. There was no in-hospital mortality. A total of 
Table 2: Risk classification of patients after initial history, physical examination and ECG, but before blood samples. Modified after [6].

\begin{tabular}{|c|c|c|c|c|c|c|c|}
\hline \multicolumn{2}{|r|}{ Risk categories } & \multirow{2}{*}{$\begin{array}{l}n \\
6\end{array}$} & \multirow{2}{*}{$\begin{array}{c}\% \text { of all } \\
4\end{array}$} & \multirow{2}{*}{$\begin{array}{l}\text { Age (yrs) } \\
78.7 \pm 3.8\end{array}$} & \multirow{2}{*}{$\begin{array}{c}\begin{array}{c}\% \text { admit- } \\
\text { ted }\end{array} \\
100\end{array}$} & \multirow{2}{*}{$\begin{array}{c}\% \text { of men } \\
6\end{array}$} & \multirow{2}{*}{$\begin{array}{c}\begin{array}{c}\% \text { of } \\
\text { women }\end{array} \\
I^{*}\end{array}$} \\
\hline I. & Obvious AMI & & & & & & \\
\hline & $\begin{array}{l}\text { Typical symptoms and ST-elevation with or with- } \\
\text { out } \mathrm{Q} \text {-waves on the ECG, or LBBB not known to } \\
\text { be old. }\end{array}$ & & & & & & \\
\hline \multirow[t]{6}{*}{ II. } & Strong suspicion of ACS & 26 & 17 & $68.6 \pm 2.2$ & 100 & 13 & 22 \\
\hline & $\begin{array}{l}\text { a) Typical symptoms without ST-elevation or Q- } \\
\text { wave }\end{array}$ & & & & & & \\
\hline & $\begin{array}{l}\text { b) Atypical symptoms with STT changes or LBBB } \\
\text { not known to be old }\end{array}$ & & & & & & \\
\hline & c) History of unstable angina regardless of ECG & & & & & & \\
\hline & $\begin{array}{l}\text { d) Acute heart failure or hypotension regardless of } \\
\text { ECG }\end{array}$ & & & & & & \\
\hline & e) VT/VF or AV-block III & & & & & & \\
\hline \multirow[t]{2}{*}{ III. } & Vague suspicion of ACS & 46 & 29 & $63.7 \pm 2.2$ & 70 & 27 & 33 \\
\hline & $\begin{array}{l}\text { Unclear symptoms and history, normal or } \\
\text { nonischemic ECG. }\end{array}$ & & & & & & \\
\hline \multirow[t]{4}{*}{ IV. } & No suspicion of ACS & 79 & 50 & $55.0 \pm 2.5$ & 13 & 54 & $43^{*}$ \\
\hline & a) No suspicion of ischemic heart disease & & & & & & \\
\hline & b) Stable angina pectoris & & & & & & \\
\hline & All included patients & 157 & 100 & $60.8 \pm 1.5$ & 47 & 100 & 100 \\
\hline
\end{tabular}

ACS, Acute coronary syndrome; LBBB, Left bundle branch block; VT/VF, ventricular tachycardia/fibrillation. *P $<0.05$ compared to men.

Table 3: Primary in-hospital destinations, PTCA interventions within 30 days of presentation and discharge diagnoses for the 74 admitted patients.

\begin{tabular}{|c|c|c|c|c|c|c|c|c|c|c|c|c|c|}
\hline \multirow{2}{*}{$\begin{array}{c}\text { Risk } \\
\text { category }\end{array}$} & \multirow[t]{2}{*}{ Admitted } & \multicolumn{4}{|c|}{ Primary in-hospital destination (\%) } & \multirow{2}{*}{$\begin{array}{c}\text { PTCA } \\
\text { (\%) }\end{array}$} & \multicolumn{7}{|c|}{ Discharge diagnoses (\%) } \\
\hline & & $w$ & ICW & $\mathrm{CICU}$ & Total & & QMI & NQMI & UA & $A P$ & $\begin{array}{c}C P \\
N U D\end{array}$ & Other & Total \\
\hline $\mathbf{I}$ & 6 & - & 17 & 83 & 100 & 17 & 67 & 33 & - & - & - & - & 100 \\
\hline II & 26 & - & 67 & 33 & 100 & 30 & - & 11 & 26 & 22 & 22 & 19 & 100 \\
\hline III & 32 & 3 & 81 & 16 & 100 & 2 & - & 6 & 6 & 9 & 34 & 44 & 100 \\
\hline IV & 10 & 50 & 50 & - & 100 & 0 & - & - & - & 10 & 40 & 50 & 100 \\
\hline All & 74 & 7 & 68 & 25 & 100 & 6 & 5 & 9 & 12 & 13 & 28 & 32 & 100 \\
\hline
\end{tabular}

W, general ward; ICW, intermediate care ward; CICU, cardiac intensive care unit; PTCA, ballon angioplasty; QMI, Q-wave myocardial infarction; NQMI, non-Q-wave Ml; UA, unstable angina pectoris; AP, stable angina pectoris; CP NUD, chest pain, unspecific.

10 patients underwent coronary angiography and 9 PTCA (table 3) within 30 days of presentation, most of them from risk category II. The PTCA rate within 30 days was $43 \%$ in the patients with NQMI or UA; FRISC II criteria [14] for early revascularisation in unstable coronary disease were generally used.

\section{Patients without ACS as the discharge diagnose}

Fifty-five patients (40\%) of 138 without ACS were admitted. Of these 55, 17 patients were from risk category II, 28 from category III, and 10 (= all admitted) patients from category IV. Compared with the 19 patients with ACS, the patients without ACS were younger, $59.3 \pm 1.7$ vs. $70.8 \pm$ 2.8 years $(\mathrm{P}<0.05)$. In risk categories II and III, however, 
patients with and without ACS were of similar age: $67.4 \pm$ 3.2 vs. $65.1 \pm 1.9$ years (n.s.). Surprisingly, at presentation $38 \%$ of all patients without ACS had pain in the left arm and only $6 \%$ of the patients with ACS $(P=0.01$, z-test $)$. In fact, in categories II and III no patients with ACS $(\mathrm{n}=14)$ had left arm pain vs. $49 \%$ in those without ACS ( $n=59$; $P$ $<0.01$, z-test). Apart from this, there was no difference between patients with and without ACS regarding presenting symptoms, risk factors or history of cardiovascular disease or diabetes.

Of the 55 patients who were admitted and did not have ACS, at least 20 (37\%) did not have any other condition or diagnosis requiring admission. These "overadmitted" patients could thus, with perfect immediate diagnostics available, have been immediately discharged from the ED and managed in an outpatient setting.

\section{Patients discharged from the emergency department}

Eighty-three patients were immediately discharged, all of them from risk categories III and IV. These patients were significantly younger than those admitted, $54.7 \pm 2.3$ vs. $67.4 \pm 1.7$ years $(\mathrm{p}<0.001)$, more seldom had previous angina pectoris ( $30 \%$ vs. $54 \%, \mathrm{P}<0.01$, z-test) or had a history of CABG or PTCA (16\% vs. 33\%, P < 0.05 , z-test). No significant difference as to presenting symptoms between admitted and discharged patients was observed. During a 6 month follow-up, none were hospitalized with $\mathrm{UA}$ or AMI and only patient died; from lung cancer. One patient underwent a CABG planned from before the index visit.

\section{Eligibility for a possible chest pain unit}

Nine patients in category II had a normal or definitely non-ischemic ECGs at presentation, and only one of them had an ACS (UA). These 9 patients, together with all 32 admitted patients in risk category III were reviewed for CPU eligibility. At presentation, $8(20 \%)$ of these 41 patients had tachy- or brady-arrhythmia, $2(5 \%)$ had rales to indicate heart failure and $9(22 \%)$ were unable to perform our standard bicycle stress test, and were therefore considered unsuitable for a CPU. A total of 29 patients $(71 \%)$ did not meet any of these exclusion criteria and would thus be eligible for a CPU.

\section{Discussion}

The present survey includes data from 157 consecutive patients presenting at the ED of Lund University Hospital during ten days. Patient characteristics, ED physicians' estimates of risk of ACS, primary destinations in the hospital and discharge diagnoses are reported. Corresponding data from Sahlgrenska Hospital in Gothenburg, Sweden $[5,6]$, and from several hospitals in the United States [7,8] have previously been reported. Judging by the number of patients with direct chest pain $(80 \%)$, the inclusion crite- ria in this study was likely broader than in the Gothenburg study [5] but similar to those in the more recent American study [7]. Accordingly, the ACS rate among those admitted seemed higher in Gothenburg, probably being about $30-40 \%$, than in the United States $(23 \%)$ and in the present investigation (26\%). Also, more patients seemed to develop an AMI in Gothenburg (at least 20\%) than in the present (15\%) and the American study (12\%), which also perhaps could be explained by the use of older and less specific markers of myocardial injury (serum aspartate aminotransferase and creatine kinase), and/or an improved in-hospital therapy for unstable angina since the Gothenburg study.

The physicians' initial suspicion of ACS seemed to be a good predictor of patient outcome, although the number of observations were small (table 3 ). As in previous investigations [6,7], only a small fraction (26\%) of those admitted were diagnosed with ACS. For instance, of those admitted to the ICW, only four patients in $51(8 \%)$ had ACS. In risk categories II and III, $63 \%$ and $88 \%$ of the admitted patients did not have ACS (table 3 ). In some cases however, hospitalization might have prevented the development of ACS. For instance, patients in risk risk category II were often treated with aspirin, betablockers, low molecular weight heparin and sometimes also clopidogrel. A surprising finding was that in risk categories II and III no patients with ACS had pain in the left arm compared to $49 \%$ in the patients without ACS. We do not have a good explanation for this. The observation challenges prevailing clinical wisdom $[15,16]$ and clearly needs to be confirmed in a larger study.

In further concert with previous surveys [6,7], a large proportion of patients were considered to be at very low risk and were immediately discharged. The discharge rate in the present study (53\%) was higher than in Gothenburg and USA (34\% in both, P $<0.001$, z-test). Most immediate dismissals were correct, since among these patients there were no cardiac events at 6 months. Previous data $[17,18]$ indicate that $2(-5) \%$ of all patients with AMI, and more with UA, are erroneously being sent home from the ED, which would here correspond to one or two patients of the 83 discharged.

To decrease unnecessary admissions, erroneous discharge and overall cost, dedicated chest pain units (CPUs) have been established in many EDs, principally in the United States $[1,2]$. Randomized controlled trials on the value of these centers are so far very few [19-21], but the body of evidence indicates $[3,4]$ that CPUs can introduce more effective patient management with a quality similar to that in traditional EDs. Cost savings have been reported per evaluated patient [3], but not yet at the hospital level. In the CPUs, patients typically undergo serial blood samples 
for markers of myocardial injury [22], serial or continuous ECG $[23,24]$, echocardiography $[25,26]$, exercise testing [27] and/or myocardial perfusion imaging [28], according to local practice. Focus in CPUs has been on the evaluation of low to moderate risk patients with a non-diagnostic ECG [29,30], which in the present study would probably correspond to all admitted patients in category III and perhaps the 9 patients in category II with normal ECGs. With commonly used exclusion criteria (Methods), almost 3/4 of these patients would be eligible for a CPU, corresponding to about 3 patients per $24 \mathrm{~h}$. The number of patients suitable for a CPU can obviously vary with patient spectrum: In an inner-city ED in Chicago [31], only about $1 / 4$ of the low-risk patients were considered eligible for a CPU protocol, albeit with the use of somewhat stricter exclusion criteria than here. For instance, $42 \%$ were unsuitable for a CPU because of inability to perform a stress test, compared to $22 \%$ in the present investigation.

Even if some of the exclusion criteria would be modified in the present analysis, the number of patients would seem to be at the lower end of what is required for a costeffective CPU. On the other hand, the current high occupancy and the high false admission rate in the ICW definitely favors the introduction of systematic risk-based accelerated diagnostic protocols in our ED. An attractive option might therefore be to supplement our conventional ED strategy with risk stratification algorithms [8,32] and selected investigations such as provocative testing or radionuclide perfusion imaging. In our ED, using the Goldman risk prediction algorithm [32] would clearly decrease CICU admissions since many of these patients did not meet the Goldman criteria for coronary care unit care: Clearly ischemic ECG and at least two of the following: Acute heart failure, hypotension or symptoms of unstable ischemic heart disease. Regarding perfusion imaging, a review of the patients in the present study suggested that in risk categories II and III, between one and two ED patients would be suitable for myocardial scintigraphy per $24 \mathrm{~h}$, using the inclusion criteria normal ECG, ongoing symptoms, no previous $\mathrm{MI}$ and planned admission solely for suspected ACS. If image results would be as expected from the observed clinical courses, almost 8 out of 10 investigated would have had negative images and be candidates for discharge from the ED; The negative predictive value of myocardial perfusion imaging can be expected to be close to $100 \%$ in this patient population [33]. Theoretically, about 15 inpatient days could then be saved during the observation period. Yet another possibility to improve ED efficiency may be to have a general practitioner in the ED to evaluate patients considered to be at very low risk of ACS by e.g. the attending nurse. Patients with very low risk were frequent in the present survey: eighty-seven percent of the patients in risk category IV, corresponding to almost
7 patients per day, were sent home from the ED with no evidence of cardiac events at 6 months.

\section{Limitations and future questions}

The present investigation includes a limited number of patients at a single hospital ED, and the results may thus not apply to other hospitals. On the other hand, consecutive patients were analyzed and few data were missing and the conclusions may therefore be reasonably valid for other hospitals of the same type. Other limitations of the present study were that seasonal variation in patient presentation was not considered, and that no serum markers of myocardial injury or ECGs were obtained after discharge. Hence, some cardiac events could have been missed in the follow-up. Whatever the case, the main results and conclusions of this investigation refer to in-patient care and outcome.

Future studies should among other things compare modern conventional ED management supplemented by risk prediction algorithms and/or selected investigations such as exercise stress testing [27] and myocardial perfusion imaging [28], with the CPU concept strategy. It may well be that improved conventional care will decrease or even eliminate the benefits of establishing dedicated chest pain units.

\section{Conclusions}

This survey indicates that despite some diagnostic advances in the ED, a large majority of patients hospitalized for suspected ACS from an ED in Sweden do not have it, and that at least 4 out of 10 of these patients can be considered "overadmitted". A comparison with older studies suggests that there has been little, if any, improvement over time. With the introduction of new and systematic risk-based diagnostic protocols, many of the now admitted patients could most likely be treated as outpatients, and a few of the admitted could probably get earlier adequate intervention. In the type of ED described in the present study, patient numbers may be too small to establish a CPU. As an alternative, traditional ED diagnostics may be supplemented with risk prediction algorithms and selected special investigations, such as myocardial scintigraphy. This type of strategy and the CPU concept care need to be compared in randomized investigations.

\section{Competing interests}

None declared.

\section{Authors' contributions}

UE conceived the study, designed the protocol, collected and analyzed data, and wrote the manuscript. H-JN performed the patient follow-up. AF collected and analyzed data. OT participated in the design of the study and reviewed the manuscript. 
All authors read and approved the final manuscript.

\section{Acknowledgements}

We are indebted to the staff at the emergency department of Lund University Hospital for help with this study.

\section{References}

I. Zalenski RJ, Rydman RJ, Ting S, Kampe L, Selker HP: A national survey of emergency department chest pain centers in the United States. Am J Cardiol 1998, 8 I : I 305-1309

2. Storrow $A B$, Gibler WB: Chest pain centers: diagnosis of acute coronary syndromes. Ann Emerg Med 2000, 35:449-46I

3. Quin G: Chest pain evaluation units. West J Med 2000, 173:403407

4. Goodacre SW: Should we establish chest pain observation units in the UK? A systematic review and critical appraisal of the literature. J Accid Emerg Med 2000, I 7: I-6

5. Karlson BW, Herlitz J, Pettersson P, Ekvall HE, Hjalmarson A: Patients admitted to emergency room with symptoms indicative of acute myocardial infarction. J Intern Med 1991, 230:25I258

6. Karlson BW, Herlitz J, Wiklund O, Richter A, Hjalmarson A: Early prediction of acute myocardial infarction from clinical history, examination and electrocardiogram in the emergency room. Am I Cardiol 199|, 68:17|-175

7. Pope JH, Ruthazer R, Beshansky JR, Griffith JL, Selker HP: Clinical Features of Emergency Department Patients Presenting with Symptoms Suggestive of Acute Cardiac Ischemia: A Multicenter Study. J Thromb Thrombolysis 1998, 6:63-74

8. Selker HP, Beshansky JR, Griffith JL, Aufderheide TP, Ballin DS, Bernard SA, Crespo SG, Feldman JA, Fish SS, Gibler WB, Kiez DA, McNutt RA, Moulton AW, Ornato JP, Podrid PJ, Pope JH, Salem DN, Sayre MR, Woolard $\mathrm{RH}$ : Use of the acute cardiac ischemia time-insensitive predictive instrument (ACI-TIPI) to assist with triage of patients with chest pain or other symptoms suggestive of acute cardiac ischemia. A multicenter, controlled clinical trial. Ann Intern Med 1998, I 29:845-855

9. Braunwald E, Antman EM, Beasley JW, Califf RM, Cheitlin MD, Hochman JS, Jones RH, Kereiakes D, Kupersmith J, Levin TN, Pepine CJ Schaeffer JW, Smith EE, Steward DE 3rd, Theroux P, Alpert JS, Eagle KA, Faxon DP, Fuster V, Gardner T], Gregoratos G, Russell RO, Smith SC Jr: ACCIAHA guidelines for the management of patients with unstable angina and non-ST-segment elevation myocardial infarction. A report of the American College of Cardiology/American Heart Association Task Force on Practice Guidelines (Committee on the Management of Patients With Unstable Angina). J Am Coll Cardiol 2000, 36:9701062

10. Giovas P, Papadoyannis D, Thomakos D, Papazachos G, Rallidis M Soulis D, Stamatopoulos C, Mavrogeni S, Katsilambros N: Transmission of electrocardiograms from a moving ambulance. J Telemed Telecare 1998, 4(Suppl I):5-7

II. Ng SM, Krishnaswamy P, Morissey R, Clopton P, Fitzgerald R, Maisel AS: Ninety-minute accelerated critical pathway for chest pain evaluation. Am / Cardiol 2001, 88:6II-6I7

12. Newby LK, Storrow AB, Gibler WB, Garvey JL, Tucker JF, Kaplan AL Schreiber DH, Tuttle RH, McNulty SE, Ohman EM: Bedside multimarker testing for risk stratification in chest pain units: The chest pain evaluation by creatine kinase-MB, myoglobin, and troponin I (CHECKMATE) study. Circulation 2001, I 03:1832 1837

13. Van der Does E, Lubsen J, Pool J, Hugenholtz PG, Arntzenius AC: Objectives and design of the IMIR study. Hart Bulletin 1976, 9I-98

14. Investigators FI: Invasive compared with non-invasive treatment in unstable coronary-artery disease: FRISC II prospective randomised multicentre study. Lancet 1999, 354:708-7| 5

15. Jesse RL, Kontos MC: Evaluation of chest pain in the emergency department. Curr Probl Cardiol 1997, 22:149-236

16. Goodacre S, Locker T, Morris F, Campbell S: How useful are clinical features in the diagnosis of acute, undifferentiated chest pain? Acad Emerg Med 2002, 9:203-208

17. Lee TH, Rouan GW, Weisberg MC, Brand DA, Acampora D, Stasiulewicz C, Walshon J, Terranova G, Gottlieb L, Goldstein-Wayne B Clinical characteristics and natural history of patients with acute myocardial infarction sent home from the emergency room. Am J Cardiol 1987, 60:219-224

18. Pope JH, Aufderheide TP, Ruthazer R, Woolard RH, Feldman JA, Beshansky JR, Griffith JL, Selker HP: Missed diagnoses of acute cardiac ischemia in the emergency department. $N$ Engl J Med 2000, 342: II63-1170

19. Farkouh ME, Smars PA, Reeder GS, Zinsmeister AR, Evans RW, Meloy TD, Kopecky SL, Allen M, Allison TG, Gibbons RJ, Gabriel SE: A clinical trial of a chest-pain observation unit for patients with unstable angina. Chest Pain Evaluation in the Emergency Room (CHEER) Investigators. N Engl J Med I998, 339: I882- I888

20. Roberts RR, Zalenski RJ, Mensah EK, Rydman RJ, Ciavarella G, Gussow L, Das K, Kampe LM, Dickover B, McDermott MF, Hart A, Straus HE, Murphy DG, Rao R: Costs of an emergency departmentbased accelerated diagnostic protocol vs hospitalization in patients with chest pain: a randomized controlled trial. JAMA 1997, 278: 1670-1676

21. Gomez MA, Anderson JL, Karagounis LA, Muhlestein JB, Mooers FB: An emergency department-based protocol for rapidly ruling out myocardial ischemia reduces hospital time and expense: results of a randomized study (ROMIO). J Am Coll Cardiol I996, 28:25-33

22. O'Neil BJ, Ross MA: Cardiac markers protocols in a chest pain observation unit. Emerg Med Clin North Am 200I, 19:67-86

23. Fesmire FM, Percy RF, Bardoner JB, Wharton DR, Calhoun FB: Usefulness of automated serial I2-lead ECG monitoring during the initial emergency department evaluation of patients with chest pain. Ann Emerg Med 1998, 3 I:3- II

24. Fesmire FM, Smith EE: Continuous I 2-lead electrocardiograph monitoring in the emergency department. Am J Emerg Med 1993, I I:54-60

25. Kontos MC, Arrowood JA, Paulsen WH, Nixon JV: Early echocardiography can predict cardiac events in emergency department patients with chest pain. Ann Emerg Med 1998, 3 I:550-557

26. Kontos MC, Kurdziel K, McQueen R, Arrowood JA, Jesse RL, Ornato $J P$, Paulsen WH, Tatum JL, Nixon JV: Comparison of 2-dimensional echocardiography and myocardial perfusion imaging for diagnosing myocardial infarction in emergency department patients. Am Heart J 2002, I 43:659-667

27. Chandra A, Rudraiah L, Zalenski RJ: Stress testing for risk stratification of patients with low to moderate probability of acute cardiac ischemia. Emerg Med Clin North Am 200I, 19:87-103

28. Abbott $B G, W$ ackers $F$ : The role of radionuclide imaging in the triage of patients with chest pain in the emergency department. Rev Port Cardiol 2000, I 9(Suppl I):153-16I

29. Wilkinson $\mathrm{K}$, Severance $\mathrm{H}$ : Identification of chest pain patients appropriate for an emergency department observation unit. Emerg Med Clin North Am 200I, I 9:35-66

30. Lee TH, Goldman L: Evaluation of the patient with acute chest pain. N Engl J Med 2000, 342: I I87-I I95

31. Zalenski RJ, Rydman RJ, McCarren M, Roberts RR, Jovanovic B, Das $\mathrm{K}$, Mensah EK, Kampe LM: rapid diagnostic protocol for an emergency department chest pain unit. Ann Emerg Med 1997, 29:99-108

32. Goldman L, Cook EF, Johnson PA, Brand DA, Rouan GW, Lee TH: Prediction of the need for intensive care in patients who come to the emergency departments with acute chest pain. N Engl J Med 1996, 334:1498-1504

33. Heller GV, Stowers SA, Hendel RC, Herman SD, Daher E, Ahlbers AW, Baron JM, Mendes dLC, Rizzo JA, Wackers FJ: Clinical value of acute rest technetium-99m tetrofosmin tomographic myocardial perfusion imaging in patients with acute chest pain and nondiagnostic electrocardiograms. J Am Coll Cardiol I998, 31:1011-1017

\section{Pre-publication history}

The pre-publication history for this paper can be accessed here:

http://www.biomedcentral.com/1471-227X/2/1/prepub 